\title{
Testi per il Giorno della Memoria
}

\section{Cristiana Facchini e Roberta Mazza (a cura di)}

Storicamente, 2 (2006).

ISSN: 1825-411X. Art. no. 1. DOI: 10.12977/stor531

Università degli Studi di Bologna

Dipartimento di Discipline Storiche

Piazza San Giovanni in Monte 2

Aula G. Prodi

Lunedì 30 gennaio, ore 17

"Nero latte dell'alba lo beviamo la sera

lo beviamo a mezzogiorno e al mattino lo beviamo la notte beviamo beviamo".

Sono i versi scarni del più noto poeta della Shoà, che ha descritto l'atroce esperienza della persecuzione nel linguaggio lirico della poesia. È Celan, ebreo rumeno che scrive in lingua tedesca, la lingua dei carnefici, il quale, contro la stentorea affermazione di Adorno, "...scrivere una poesia dopo Auschwitz è un atto di barbarie, e ciò avvelena la stessa consapevolezza del perché è divenuto impossibile scrivere oggi poesie...", sperimenta, con risultati altissimi, la potenzialità del linguaggio lirico di mettere in essere, di fare sentire, la Shoà.

Aharon Appelfeld, scrittore israeliano di origine rumena, sopravvissuto ai campi, trae spunto proprio dagli stessi versi della poesia di Celan, rispondendo ad una domanda rivoltagli da Philip Roth. Dice, parlando dei sopravvissuti, dei superstiti, che "il sopravvissuto ... ha ingoiato l'Olocausto tutto intero, e procede nella vita con l'Olocausto in tutte le sue membra. Beve il "latte nero" del poeta Celan, mattino, pomeriggio e sera. Non ha 
alcun vantaggio su nessun altro, ma non ha ancora perduto il suo volto umano".

Più ironico e solare, Primo Levi, scrittore non "professionista", autore del capolavoro Se questo è un uomo, nel suo libro Se non ora quando?, dedicato agli ebrei dell'Europa orientale, propende per un percorso narrativo più lieve. In questo libro, il cui titolo è tratto da un antico insegnamento rabbinico, Levi confessa che voleva "raccontare una storia piena di speranza, a tratti allegra, benché proiettata su uno scenario sanguinoso. Volevo combattere un luogo comune ancora prevalente in Italia: un ebreo è una persona mite, uno studioso, inadatto alla guerra, umiliato che ha sopportato secoli di persecuzione senza mai reagire. Mi sembrava doveroso rendere omaggio a quegli ebrei che, in condizioni disperate, trovarono il coraggio e la capacità di resistere".

I diversi registri stilistici e narrativi con cui ebrei e non ebrei hanno espresso l'esperienza traumatica del fascismo, del nazismo, delle persecuzioni e della guerra vi vengono presentati in un percorso di letture.

Abbiamo deciso di ricordare attraverso i brani letterari, poesie e lettere, stralci di testi narrativi, riflessioni teoriche e filosofiche di coloro che hanno vissuto questo momento storico. Abbiamo scelto le parole di autori noti e meno noti, poeti, scrittori, filosofi, storici, ma anche comuni cittadini.

La memoria, restituita dai brani che presentiamo, non passa solo attraverso i versi lirici che fissano e restituiscono l'esperienza del trauma e della perdita, ma è fatta anche delle voci speranzose di coloro che sono stati perseguitati, per motivi politici, razziali e religiosi, dai regimi nazi-fascisti.

Voci che invitano ad una riflessione sul dovere dell'azione e del ricordo, ma incitano anche alla speranza irrinunciabile di un mondo migliore.

Questa lettura deve molto al lavoro di Riccardo Bonavita e in parte anche a lui è dedicata. 
Cristiana Facchini

Roberta Mazza

\section{SHEMÀ}

[[figure

align="left"]]figures/2006/mazza_shoa/mazza_shoa_2006_01.jpg[[/figure]]

Voi che vivete sicuri

Nelle vostre tiepide case

Voi che trovate tornando a sera

Il cibo caldo e visi amici:

Considerate se questo è un uomo,

Che lavora nel fango

Che non conosce pace

Che lotta per mezzo pane

Che muore per un sì o per un no.

Considerate se questa è una donna,

Senza capelli e senza nome

Senza più forza di ricordare

Vuoti gli occhi e freddo il grembo

Come una rana d'inverno.

Meditate che questo è stato:

Vi comando queste parole.

Scolpitele nel vostro cuore

Stando in casa andando per via,

Coricandovi alzandovi:

Ripetetele ai vostri figli.

O vi si sfaccia la casa,

La malattia vi impedisca,

I vostri nati torcano il viso da voi. 
Primo Levi, Se questo è un uomo, Torino, Einaudi 1956

\section{Il Testamento di Marc Bloch}

[[figure

align="left"]]figures/2006/mazza_shoa/mazza_shoa_2006_02.jpg[[/figure]]

Clermont-Ferrand, 18 marzo 1941

Dovunque io muoia, in Francia o in terra straniera, e in qualsiasi momento ciò accada, lascio alla mia cara moglie o in sua mancanza ai miei figli la cura di provvedere ai miei funerali, come riterranno opportuno. Saranno funerali puramente civili: i miei cari sanno che non ne avrei voluti di diversi. Ma spero che quel giorno - nella camera ardente o al cimitero - un amico voglia dar lettura di queste poche parole:

Non ho chiesto che sulla mia tomba si recitassero le preghiere ebraiche la cui cadenza, purtuttavia, accompagnò all'ultimo riposo tanti miei antenati e il mio stesso padre. Per tutta la vita, come meglio ho potuto, ho teso a una totale sincerità d'espressione e di spirito. Ritengo la compiacenza alla menzogna, qualunque sia il pretesto che essa accampi, la peggior lebbra dell'animo. Come qualcuno tanto più grande di me, desidererei che la mia tomba, quale unico motto, portasse incise queste semplici parole: Dilexit veritatem. Per questo non potevo accettare che in quest'ora di supremi addii, quando ogni uomo ha il dovere di riassumere se stesso, si invocasse in mio nome l'ardore di una ortodossia di cui non riconosco il credo. 
Ma ancora più odioso sarebbe per me se vi fosse chi, in questo atto di onestà, ravvisasse qualcosa di simile ad un vile rinnegamento. Affermo dunque se è necessario di fronte alla morte di essere nato ebreo; che mai ho pensato di negarlo, né mai ho avuto motivo di essere tentato di farlo. In un mondo assalito dalla più atroce barbarie, la generosa tradizione dei profeti ebrei, che il cristianesimo, in ciò che ebbe di più puro, riprese e diffuse, non resta forse una delle nostre migliori ragioni per vivere, credere, lottare?

Estraneo ad ogni formalismo confessionale come ad ogni presunta solidarietà razziale, per tutta la vita mi sono sentito anzitutto e semplicemente francese. Legato alla mia patria da una già lunga tradizione famigliare, nutrito della sua eredità spirituale e della sua storia, incapace, in verità, di concepirne un'altra in cui respirare a pieni polmoni, l'ho amata molto e servita con tutte le mie forze. Mai il mio essere ebreo mi è parso di ostacolo a questi sentimenti. Nel corso di due guerre, non mi è stato dato di morire per la Francia. Almeno, che io possa rendere a me stesso questa testimonianza: muoio, come ho vissuto, da buon francese.

Poi - se sarà possibile prodursene il testo - si dia lettura delle mie cinque citazioni.

Marc Bloch, La strana disfatta. Testimonianza del 1940, Einaudi 1995

Nelly Sachs

Coro dei superstiti

[[figure

align="left"]]figures/2006/mazza_shoa/mazza_shoa_2006_03.jpg[[/figure]] Noi superstiti dalle nostre ossa la morte ha già intagliato i suoi flauti, sui nostri tendini ha già passato il suo archetto -

I nostri corpi ancora si lamentano col loro canto mozzato. 
Noi superstiti

davanti a noi, nell'aria azzurra, pendono ancora i lacci attorti per i nostri colli -

le clessidre si riempiono ancora con il nostro sangue.

Noi superstiti,

ancora divorati dai vermi dell'angoscia -

la nostra stella è sepolta nella polvere.

Noi superstiti

vi preghiamo:

mostrateci lentamente il vostro sole.

Guidateci piano di stella in stella.

Fateci di nuovo imparare la vita.

Altrimenti il canto di un uccello,

il secchio che si colma alla fontana

potrebbero far prorompere il dolore

a stento sigillato

e farci schiumar via -

Vi preghiamo:

non mostrateci ancora un cane che morde

potrebbe darsi, potrebbe darsi

che ci disfiamo in polvere

davanti ai vostri occhi.

Ma cosa tiene unita la nostra trama?

Noi, ormai senza respiro,

la nostra anima è volata a lui dalla mezzanotte

molto prima che il nostro corpo si salvasse

nell'arca dell'istante -

Noi superstiti,

stringiamo la vostra mano,

riconosciamo i vostri occhi -

ma solo l'addio ci tiene ancora uniti,

l'addio nella polvere 
ci tiene uniti a voi -

Nelly Sachs, Opere scelte, in Shemeul Josef Agnon - Nelly Sachs, Opere, Torino, Utet, 1996

Sogni

(adattamento da Charlotte Berardt, Il Terzo Reich dei Sogni, Einaudi 1991 e Aharon Appelfeld, intervista con Philip Roth, in Chiacchiere di Bottega, Einaudi Torino, 2004)

[[figure

align="left"]]figures/2006/mazza_shoa/mazza_shoa_2006_04.jpg[[/figure]]

Giobbe disse: "Nei fantasmi, tra visioni notturne, quando grava sugli uomini il sonno, terrore mi prese e spavento, e tutte le ossa mi fece tremare". "Macbeth ha ucciso il sonno!". II terzo Reich ha ucciso il sonno, si insinua nei sogni. Come I'Inquisizione vigila e colpisce chi ha "pronunciato eresie in sogno".

Ascolta una giovane donna, nell'estate del 1933:

"Sogno di parlare russo in sogno - una lingua che non conosco, e del resto non parlo nel sonno -, come misura precauzionale, affinché non capisca me stessa e nessuno possa capirmi, in caso mi sfugga qualcosa a proposito dello stato, visto che ciò è proibito e va denunciato"

II Reich controlla la lingua, e nello spazio intimo della persona impone immagini kafkiane, L'ideologia plasma i contorni e le forme della realtà:

"Sono cresciuto con la sensazione che tutto ciò che era ebreo fosse deforme. Fin dalla più tenera età il mio sguardo si è rivolto alla bellezza dei non ebrei. Erano alti e biondi e si comportavano con naturalezza". 
"Una ragazza di ventidue anni, con un naso sottile, ma piuttosto adunco, che ne domina il volto, crede evidentemente che ormai tutti la prendano per ebrea. Nasi e documenti, documenti e nasi incominciano a popolare i suoi sogni".

"Mi presento all'ufficio per la documentazione sull'appartenenza alla razza ariana [.] per esibire un certificato, relativo a mia nonna, che mie era costato mesi di ricerca. L'impiegato, che ha l'aspetto di una statua di marmo e siede dietro un muro, allunga un braccio oltre questo muro, afferra il certificato, lo straccia a pezzetti, poi brucia tutto in una stufa incassata nel muro: - E adesso sei sempre di pura razza ariana?".

\section{Paul Celan}

Fuga di morte

\section{[[figure}

align="left"]]figures/2006/mazza_shoa/mazza_shoa_2006_05.jpg[[/figure]]

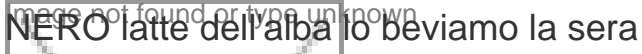

o beviamo a mezzogiorno e al mattino lo beviamo la notte

beviamo e beviamo

scaviamo una tomba nell'aria là non si giace stretti.

Nella casa abita un yomo che gioca con i serpenti che scrive che scrive all'imbrunire in Germania i tuoi capelli d'oro Margarete o scrive ed esce dinanzi a casa e brillano le stelle e fischia ai suoi mastini fischia ai suoi ebrei e fa scavare una tomba nella terra ci comanda ora suonate alla danza

Nero latte dell'alba ti beviamo la notte

ti beviamo al mattino e a mezzogiorno ti beviamo la sera beviamo e beviamo

Nella casa abita un uomo che gioca con i serpenti che scrive che scrive all'imbrunire in Germania i tuoi capelli d'oro Margarete I tuoi capelli di cenere Sulamith scaviamo una tomba nell'aria là non si giace 
stretti

Lui grida vangate più a fondo il terreno voi e voi cantate e suonate impugna il ferro alla cintura lo brandisce i suoi occhi sono azzurri spingete più a fondo le vanghe voi e voi continuate a suonare alla danza

Nero latte dell'alba ti beviamo la notte ti beviamo a mezzogiorno e al mattino ti beviamo la sera beviamo e beviamo nella casa abita un uomo i tuoi capelli d'oro Margarete i tuoi capelli di cenere Sulamith lui gioca con i serpenti

Lui grida suonate più dolce la morte la morte è un maestro tedesco lui grida suonate più cupo i violini e salirete come fumo nell'aria e avrete una tomba nelle nubi là non si giace stretti

Nero latte dell'alba ti beviamo la notte ti beviamo a mezzogiorno la morte è un maestro tedesco ti beviamo la sera e la mattina beviamo e beviamo la morte è un maestro tedesco il suo occhio è azzurro ti colpisce con palla di piombo ti colpisce preciso nella casa abita un uomo i tuoi capelli d'oro Margarete aizza i suoi mastini contro di noi ci regala una tomba nell'aria gioca con i serpenti e sogna la morte è un maestro tedesco

i tuoi capelli d'oro Margarete i tuoi capelli di cenere Sulamith

Paul Celan, Poesie, (a cura di Giuseppe Bevilacqua), Milano, Mondadori, 1998

\section{Lo scempio del mondo}

[[figure

align="left"]]figures/2006/mazza_shoa/mazza_shoa_2006_06.jpg[[/figure]] 
Quali saranno le prospettive di risanamento della nostra civiltà quando un giorno questa guerra sarà finita per esaurimento delle potenze soccombenti sia nel lontano Oriente sia qui in Occidente?

Prospettive di risanamento: non si può dire di più. E' chiaro infatti che questo amarissimo secolo, il quale si avvicina alla sua metà in un'agonia senza esempi, reca l'impronta di una progressiva decadenza culturale che può terminare con una catastrofica distruzione.

Con ciò non vogliamo affatto negare che il secolo $X X$ abbia dato prodotti eccellenti, e nuovi e preziosi contributi alle civiltà per l'epoca presente e per l'avvenire. Rimane però l'inevitabile e deprimente quesito: dopo la fine di tanti orrori questo mondo ferito e mutilato sarà tosto capace di avere una fioritura di pura e nobile civiltà?

Le pagine che seguono contengono la conclusione che le premesse di una tale rapida rinascita culturale esisteranno solo in misura molto esigua, sicché le prospettive di una guarigione della civiltà sono paurosamente piccole. Tuttavia l'ultima parola dev'essere questa: noi non vogliamo abbandonare la speranza d'un miglioramento né la volontà di attuarlo. L'umanità non può rinunciare a quel preziosissimo retaggio che chiamiamo civiltà.

Johan Huizinga, Lo scempio del mondo, Bruno Mondadori, Milano, 2004

\section{Vittorio Sereni}

\section{NEL VERO ANNO ZERO}

\section{[[figure}

align="left"]]figures/2006/mazza_shoa/mazza_shoa_2006_07.jpg[[/figure]] Meno male lui disse, il più festante: che meno male c'erano tutti.

Tutti alle case dei Sassoni - rifacendo la conta.

Mai stato in Sachsenhausen? Mai stato.

A mangiare ginocchio di porco? Mai stato.

Ma certo, alle case dei Sassoni.

Alle case dei Sassoni, in Sachsenhausen, cosa c'è di strano? 
Ma quante Sachsenhausen in Germania, quante case.

Dei Sassoni, dice rassicurante

caso mai svicolasse tra le nebbie

un'ombra di recluso nel suo gabbano.

No non c'ero mai stato in Sachsenhausen.

E gli altri allora - mi legge nel pensiero -

quegli altri carponi fuori da Stalingrado

mummie di già soldati

dentro quel sole di sciagura fermo

sui loro anni aquilonari. dopo tanti anni

non è la stessa cosa?

Tutto ingoiano le nuove belve, tutto -

si mangiano cuore e memoria queste belve onnivore.

A balzi nel chiaro di luna si infilano in un night.

Vittorio Sereni, Poesie, (edizione critica a cura di Dante Isella), Milano,

Mondadori, 1995

\section{Lettere al muro}

Lettere di condannati a morte della Resistenza europea, Einaudi, Torino, 1995

[[figure

align="left"]]figures/2006/mazza_shoa/mazza_shoa_2006_08.jpg[[/figure]] "Non piangetemi, non chiamatemi povero. Muoio per aver servito un'idea."

(Guglielmo Jervis)

"Mimma cara,

la tua mamma se ne va pensandoti e amandoti, mia creatura adorata, sii buona, studia ed ubbidisci sempre gli zii che t'allevano, amali come fossi io. lo sono tranquilla. Tu devi dire a tutti i nostri cari parenti, nonna e gli altri, che mi perdonino il dolore che do loro. Non devi piangere né vergognarti per 
me. Quando sarai grande capirai meglio. Ti chiedo una cosa sola: studia, io ti proteggerò dal cielo"

(Paola Garelli, Mirka)

"Mia adorata Pally,

sono gli ultimi istanti della mia vita. Pally adorata ti dico a te saluta e bacia tutti quelli che mi ricorderanno. Credimi non ho mai fatto nessuna cosa che potesse offendere il nostro nome. Ho sentito il richiamo della patria per la quale ho combattuto, ora sono qui. fra poco non sarò più, muoio sicura di aver fatto quanto mi era possibile affinché la libertà trionfasse.

(Irma Marchiani, Anty)

"Musicisti e scrittori dobbiamo rinunciare ai nostri privilegi. [.] L'idea di andare a fare il partigiano in questa stagione mi diverte pochissimo; [.] Tuttavia è l'unica possibilità aperta e l'accolgo".

"Se non dovessi tornare non mostratevi inconsolabili. Una delle poche certezze acquistate nella mia esperienza è che non ci sono individui insostituibili e perdite irreparabili. Un uomo vivo trova sempre ragioni sufficienti di gioia negli altri uomini vivi, e tu che sei giovane e vitale hai il dovere di lasciare che i morti seppelliscano i morti"

(Giaime Pintor, Doppio Diario, Einaudi, Torino, 1975)

\section{Louis Borges}

\section{Giusti}

Un uomo che coltiva il suo giardino, come voleva Voltaire.

Chi è contento che sulla terra esista la musica.

Chi scopre con piacere una etimologia.

Due impiegati che in un caffè del Sud giocano in silenzio agli scacchi.

Il ceramista che intuisce un colore e una forma.

Il tipografo che compone bene questa pagina che forse non gli piace.

Una donna e un uomo che leggono le terzine finali di un certo canto. 
Chi accarezza un animale addormentato.

Chi giustifica o vuole giustificare un male che gli hanno fatto.

Chi è contento che sulla terra ci sia Stevenson.

Chi preferisce che abbiano ragione gli altri.

Tali persone, che si ignorano, stanno salvando il mondo.

(Louis Borges)

Vedi anche:

Poesie sulla Shoah 